- Editorial •

Edtor's Focus
April 2022 Vol. 65 No. 4: 240361

https://doi.org/10.1007/s11433-022-1866-5

\title{
Interfacing superconducting and atomic qubits via unconventional geometric quantum operations
}

Interfacing or entanglement between spatially separated quantum systems, such as superconducting qubits, semiconductor quantum dots, atomic qubits, and solid-state color centers, is crucial in quantum networks and distributed quantum computation [1]. Significant progress has been made in generating entanglement between spatially separated quantum systems via optical links [2], while remote superconducting chips, whose quantum states are easily spoiled by optical photons, have been entangled via cryogenic microwave links [3].

Cold neutral atoms can be used to construct a quantum transducer capable of converting quantum signals from the microwave domain to the optical domain because they have optically and microwave addressable transitions, which are crucial requirements for quantum information technology. Furthermore, cold atoms confined near a superconducting chip have a long coherence time [4]. Interfacing superconducting qubits and atomic qubits would thus concurrently provide a quantum memory and an interface to long-range optical quantum networks for the superconducting qubits [5]. However, the strong optical lasers that are often used for microwave-optical conversion inevitably heat up the superconducting qubits and cryogenic microwave links. In a recent study, Liang et al. [6] from the South China Normal University proposed a built-in fault-tolerant geometric operation [7] to tackle this main challenge without requiring any auxiliary cooling system. First, they proposed that the two-qubit systems (superconducting and atomic qubits) be spatially separated but connected via a 1-m microwave link to prevent disturbing the superconducting qubits with lasers. Second, to suppress the detrimental effects of the thermal links, an unconventional geometric quantum operation [8] is used to generate high-fidelity entanglement between the qubits, which is naturally immune to thermal excitations (a dominant source of infidelity) $[9,10]$. Finally, the microwave link between these two qubits is placed in a $1-\mathrm{K}$ environment, which may improve the microwave link's lifetime and allow for greater cooling power. With realistic experimental settings, numerical simulations of the related master equation show that a high-concurrency entanglement between superconducting qubits and Rydberg-atom qubits may be created.

The proposed approach not only overcomes the main challenges for interfacing superconducting and Rydberg-atom qubits but also can be adopted to scale various quantum nodes via thermal links, and both interfacing and scaling are significantly important in current quantum technologies. Interestingly, the current geometric operations could be used experimentally in the near future to entangle superconducting qubits in different dilution refrigerators using thermal microwave links, noted that remote superconducting chips have been entangled using cryogenic microwave links. Entanglement between superconducting qubits and Rydberg-atom qubits would be the first step in constructing a quantum network based on nodes having superconducting quantum computers.

Gui-Lu Long

Department of Physics, Tsinghua University, Beijing 100084, China

1 D. Awschalom, K. K. Berggren, H. Bernien, S. Bhave, L. D. Carr, P. Davids, S. E. Economou, D. Englund, A. Faraon, M. Fejer, S. Guha, M. V. Gustafsson, E. Hu, L. Jiang, J. Kim, B. Korzh, P. Kumar, P. G. Kwiat, M. Lončar, M. D. Lukin, D. A. B. Miller, C. Monroe, S. W. Nam, P. Narang, J. S. Orcutt, M. G. Raymer, A. H. Safavi-Naeini, M.
Spiropulu, K. Srinivasan, S. Sun, J. Vučković, E. Waks, R. Walsworth, A. M. Weiner, and Z. Zhang, PRX Quantum 2, 017002 (2021).

2 S. Daiss, S. Langenfeld, S. Welte, E. Distante, P. Thomas, L. Hartung, O. Morin, and G. Rempe, Science 371, 614 (2021), arXiv: 2103.13095. 3 Y. Zhong, H. S. Chang, A. Bienfait, É. Dumur, M. H. Chou, C. R. Con- 
ner, J. Grebel, R. G. Povey, H. Yan, D. I. Schuster, and A. N. Cleland, Nature 590, 571 (2021), arXiv: 2011.13108.

4 S. Bernon, H. Hattermann, D. Bothner, M. Knufinke, P. Weiss, F. Jessen, D. Cano, M. Kemmler, R. Kleiner, D. Koelle, and J. Fortágh, Nat. Commun. 4, 2380 (2013), arXiv: 1302.6610.

5 X. Han, W. Fu, C. L. Zou, L. Jiang, and H. X. Tang, Optica 8, 1050 (2021).

6 Z.-T. Liang, G.-Q. Zhang, J. Yuan, Q. Ye, K. Liao, Z.-Y. Xue, H. Yan, and S.-L. Zhu, Sci. China-Phys. Mech. Astron. 65, 240362 (2022)
7 J. Zhang, T. H. Kyaw, S. Filipp, L. C. Kwek, E. Sjöqvist, and D. Tong, arXiv: 2110.03602 .

8 S. L. Zhu, and Z. D. Wang, Phys. Rev. Lett. 91, 187902 (2003), arXiv: quant-ph/0306166.

9 A. Sørensen, and K. Mølmer, Phys. Rev. A 62, 022311 (2000), arXiv: quant-ph/0002024.

10 D. Leibfried, B. DeMarco, V. Meyer, D. Lucas, M. Barrett, J. Britton, W. M. Itano, B. Jelenković, C. Langer, T. Rosenband, and D. J. Wineland, Nature 422, 412 (2003). 